\title{
Evaluation of the Impact of Calibration of Patient-Reported Outcomes Measures on Clinical Trial Results: A Simulation Study based on Rasch Measurement Theory
}

Angély Loubert ( $\square$ angely.loubert@modusoutcomes.com )

Modus Outcomes

Antoine Regnault

Modus Outcomes

Véronique Sébille

University of Nantes

Jean-Benoit Hardouin

University of Nantes

\section{Research Article}

Keywords: Clinical Trials, Patient-Reported Outcomes, Rasch Measurement Theory, Calibration

Posted Date: December 22nd, 2021

DOI: https://doi.org/10.21203/rs.3.rs-1182025/v1

License: (c) (i) This work is licensed under a Creative Commons Attribution 4.0 International License. Read Full License 


\section{Abstract}

\section{Background}

In the analysis of clinical trial endpoints, calibration of patient-reported outcomes (PRO) instruments ensures that resulting "scores" represent the same quantity of the measured concept between applications. Rasch measurement theory (RMT) is a psychometric approach that guarantees algebraic separation of person and item parameter estimates, allowing formal calibration of PRO instruments. In the RMT framework, calibration is performed using the item parameter estimates obtained from a previous "calibration" study. But if calibration is based on poorly estimated item parameters (e.g., because the sample size of the calibration sample was low), this may hamper the ability to detect a treatment effect, and direct estimation of item parameters from the trial data (non-calibration) may then be preferred. The objective of this simulation study was to assess the impact of calibration on the comparison of PRO results between treatment groups, using different analysis methods.

\section{Methods}

PRO results were simulated following a polytomous Rasch model, for a calibration and a trial sample. Scenarios included varying sample sizes, with instrument of varying number of items and modalities, and varying item parameters distributions. Different treatment effect sizes and distributions of the two patient samples were also explored. Comparison of treatment groups was performed using different methods based on a random effect Rasch model. Calibrated and non-calibrated approaches were compared based on type-l error, power, bias, and variance of the estimates for the difference between groups.

\section{Results}

There was no impact of the calibration approach on type-I error, power, bias, and dispersion of the estimates. Among other findings, mistargeting between the PRO instrument and patients from the trial sample (regarding the level of measured concept) resulted in a lower power and higher position bias than appropriate targeting.

\section{Conclusions}

Calibration of PROs in clinical trials does not compromise the ability to accurately assess a treatment effect and is essential to properly interpret PRO results. Given its important added value, calibration should thus always be performed when a PRO instrument is used as an endpoint in a clinical trial, in the RMT framework.

\section{Background}

Patient-Reported Outcomes (PRO) are defined as "any report of the status of a patient's health condition that comes directly from the patient". [1] PRO instruments are typically questionnaires for which the responses of patients to a set of items (questions) lead to the calculation of scores that are used to measure unobservable variables (also known as latent traits), such as pain, fatigue or anxiety. PRO scores are increasingly used as key endpoints to demonstrate the efficacy of new treatments in clinical trials. [2]

Clinical trials are conducted in a high stakes decision-making context. Hence, they must apply methods that warrant achieving optimal measurement quality. [3-5] Metrology defines the good scientific principles for measurement, which are widely applied in various industries. Clinical trials can be seen in the metrology framework as measuring systems of treatment effect. [6] As such, it is possible to apply the principles of metrology to the clinical trial setting, and to the measuring instruments included, such as PRO instruments. A key concept for metrology is traceability, which is defined as the "property of a measurement result whereby the result can be related to a reference through a documented unbroken chain of calibrations, each contributing to the measurement uncertainty". [6] Applying this concept to PROs in a clinical trial involves that it is possible to relate the results from the trial to those of any application of the same instrument in other contexts, by a documented chain of calibrations. [4, 7] In practice, calibration cannot relate to proper measurement standards, which do not exist for PROs. Instead, calibration for PRO instruments can be based on the results of a reference application of the PRO instrument in a sample of reference, either from a dedicated calibration study or a psychometric "validation study" of the instrument. Calibration ensures that a same PRO "score" consistently represent the same quantity of the latent variable between applications, which is essential to interpret and compare trial results. While calibration is primarily used for the measurement of physical quantities, it also plays an important role in other human sciences. For example, in education science, calibration ensures that scores from major educational tests, such as the Scholastic Aptitude Test (SAT), are calculated the same way and lead to comparable scores between each student. [8]

The question of calibration of PRO instruments became more critical with the growing use of recent psychometric methods. PRO instruments used in clinical trials were initially developed in the classical test theory (CTT) paradigm [9], where the measurement result was obtained by a raw sum score. Raw scores do not need estimates from any specific sample to be calculated, so they are calibrated by construction. But, as this approach presents several theoretical limitations [10], alternative psychometric approaches ("modern psychometric methods") are increasingly being preferred over CTT for the evaluation of PROs. Rasch Measurement Theory (RMT) is one such approach. Based on the Rasch model, it offers a different framework for calibration. The Rasch model separates the parameters of interest in the process of measurement of latent traits: item parameters ("difficulty" of the 
items, i.e., whether they discriminate more or less severe patients regarding their latent trait) and person parameters (measurements of the patient latent traits). [11-13] This property ensures independence between the sample and the instrument ("specific objectivity"), and thus, allows proper calibration (i.e., estimation of item parameters that are independent from the samples on which they have been obtained).

Considering the RMT framework, calibration of PRO instrument first requires performing an RMT analysis on data from a "calibration" sample of patients. Obtained estimates of item parameters are then set to fixed in a formal RMT analysis of the clinical trial. A similar process can be used for calibration based on Item Response Theory (IRT) models, the other paradigm of "modern psychometrics", in which additional parameters are estimated for each model. However, IRT models do not have specific objectivity and item parameters estimates are thus dependent on the sample of patients used. This is especially problematic as in calibration the obtained item parameter values aim at being generalized to different patient samples. Nevertheless, several PRO instruments developed in the RMT or IRT paradigms are used in clinical trials, with existing calibration solutions, such as the BREAST-Q [14], the Rasch-built Overall Disability Scale (R-ODS) [15], instruments from the PRO Measurement Information System (PROMIS) [16].

But, despite its major advantage for the interpretability of the PRO results, calibration might also show some negative impact. In particular, if the sample size and heterogeneity of the calibration sample is not sufficient, with patients very different from those expected from the clinical trial regarding the concept of interest (e.g., more severe symptoms), some item parameters values to be used for calibration might be misspecified. In such cases, directly running the Rasch model on the trial sample (without a preliminary calibration step) could lead to more precise estimations of item parameters that are specifically targeted to the patients included. This in turn might lead to better conditions for evaluating treatment effect. In a comparative trial, the impact of calibration might also differ depending on the method used for comparison of treatment groups. A possibility is to use a random effect Rasch model, directly including a covariate for group effect or first estimating the latent traits of the patients before performing a t-test, $[17,18]$ and the best approach still needs to be identified.

Previous simulation studies explored to some extent the impact of calibration on clinical trial results. [19, 20] However, calibration was not the main focus of these studies, and the impact of the characteristics of the calibration sample and its differences with the clinical trial sample were not evaluated. Also, these studies only explored the case where PRO instruments included only dichotomous items (with only two possible response options), which is not the most common structure for a PRO instrument in health studies.

The objective of this research was to further explore the impact of calibration on the comparison of PRO instruments between treatment groups from a clinical trial. For this purpose, we conducted a simulation study aiming to compare the use of calibrated and non-calibrated approaches on simulated polytomous PRO data from a clinical trial. The impact of calibration was assessed for two different cross-sectional analysis methods and for different characteristics of the PRO instrument and of the samples of patients used in the calibration process.

\section{Methods}

The Rasch model

The Rasch model is a measurement, probabilistic model used to measure unobserved latent traits based on observed responses to items from a questionnaire (PRO instrument). [21] The polytomous Rasch model (Partial Credit Model, PCM) is the generalization of the original Rasch model for ordered polytomous data (i.e. with more than 2, ordered, response options, of the Likert-scale type). [22] Considering a PRO instrument including J items with the same number of response options $M$ (modalities, coded from 0 to $M-1$ ) the model can be written as follows:

$$
P\left(X_{i j}=k \mid \theta_{i}, \delta_{j}\right)=\frac{\exp \left(k \theta_{i}-\sum_{l=1}^{k} \delta_{j l}\right)}{\sum_{r=0}^{M-1} \exp \left(r \theta_{i}-\sum_{l=1}^{r} \delta_{j l}\right)}(1)
$$

Where $\mathrm{k}$ is the response to patient $i(\mathrm{i}=1, \ldots, \mathrm{N})$ to item $j(\mathrm{j}=1, \ldots, \mathrm{J})$, realization of the random variable $X_{i j}(k \in\{0, \ldots M-1\}), \theta_{i}$ the latent trait for patient $i$, and $\delta_{j}$ the vector of dimension M-1 containing all category thresholds parameters $\delta_{j l}$ associated to categories $l(l=1, \ldots, M-1)$ of items $j$

Considering the patient latent traits as realizations of a random variable assumed to be normally distributed results in a random effect PCM. Since the objective of a clinical trial is to compare treatments, a corresponding group covariate for treatment effect can be added to the model. [17] Denoting $\gamma$ the parameter for the treatment effect (mean difference in latent trait between placebo and treated groups), patient latent traits are thus decomposed into a group effect $\left(\mu_{0}+g_{i} \gamma\right)$ and an individual effect $\left(\theta_{\text {res }}\right)$. The random effect PCM with treatment group effect can then be written as:

$$
P\left(X_{i j}=k \mid \mu_{0}, \gamma, \theta_{\text {res }_{i}} \delta_{j}\right)=\frac{\exp \left(k\left(\mu_{0}+g_{i} \gamma+\theta_{r e s_{i}}-\delta_{j l}\right)\right)}{\sum_{r=0}^{M} \exp \left(r\left(\mu_{0}+g_{i} \gamma+\theta_{r e s_{i}}-\sum_{l=1}^{r} \delta_{j l}\right)\right)}(2)
$$

With $g_{i}=0$ if patient $\mathrm{i}$ is in the placebo group, and $g_{i}=1$ in the treated group, and thus $\mu_{0}$ corresponding to the mean of latent traits in the placebo group. 
Patient responses to multi-item PRO instruments with polytomous responses were generated using Monte Carlo simulations with a random effect PCM. [22] This assumes that the simulated PRO instrument was previously validated with RMT. For each iteration, we generated two samples:

- One for a calibration (or validation) study of the PRO instrument.

- One for a two parallel groups (treatment vs. placebo group) clinical trial, at a post treatment occasion (cross-sectional data).

Calibration and trial samples shared the same PRO instrument characteristics, which varied based on several parameters between different scenarios:

- The number of items $\mathrm{J}$ from the PRO instrument varied between 4 and 10 , in accordance with the size of the subscales of PRO instruments that are commonly used in clinical research.

- The number of response categories $\mathrm{M}$ was of 3 or 5 , in accordance with commonly encountered number of possible response options in PRO instruments with items of the Likert-scale type (ordered response options). Response categories were coded from 0 to M-1.

- Distribution of the thresholds, $\delta_{j l}$ (which corresponds to the level of latent trait for which an patient has the same probability to endorse one or the other of two subsequent ordered response categories, with $l$ the response option, from 1 to $M-1$, of the item $j$ ) and associated item locations $\delta_{j}$ (which corresponds to the mean of the category thresholds for each given item) was designed to reflect two typical archetypes of PRO instruments encountered in practice (see Figure 1 for an illustration of the two cases):

- A first archetype where the item locations $\delta_{j}$ had a low dispersion on the continuum measured by the instrument ( $\delta_{j}$ regularly spaced from -0.25 to 0.25 ), with highly dispersed category thresholds $\delta_{j l}$ regularly spaced for one given item, based on the percentiles of a normal distribution (if the items have 3 response categories, thresholds were set to the 33rd and 66th percentiles of the distribution; If the items have 5 response categories, thresholds were set to the 20th, 40th, 60th and 80th percentiles) centered on $\delta_{j}$ with a standard deviation (SD) of 2.5. This is typically observed with instruments in which the variability over the latent trait is supposed to be captured by varying levels of the response scale. Such item distributions can be observed with instruments developed using CTT methods, as "redundancy" of the items on the continuum (items with very close category thresholds $\delta_{j l}$ ) is not identified as problematic using CTT methods [23] (in fact this pattern reflects the theoretical notion of "parallel items sets' of the CTT paradigm [24]).

- A second archetype where the item locations $\delta_{j}$ were highly dispersed on the continuum measured by the instrument $\left(\delta_{j}\right.$ regularly spaced from -1 to 1), with response category thresholds $\delta_{j l}$ with low dispersion, regularly spaced for one given item, based on the percentiles of a normal distribution centered on $\delta_{j}$ with a SD of 1.5 . This corresponds to PRO instruments in which the variability over the latent trait is supposed to be captured with items representing different levels on the continuum ("item hierarchy"). It is commonly observed with instrument developed using RMT. $[14,15]$

- The mean of item parameters was set to 0 (following the specified distribution for item parameters).

Calibration samples varied between scenarios based on several parameters:

- The full sample size of the calibration sample $N_{\text {calibration }}$ varied between 100 and 500 . Values were selected to reflect the range of sample sizes that can be encountered in clinical research studies for validation of PRO instruments. [14, 15, 25]

- The latent trait distribution was defined as normal, in line with the hypothesis underlying the use of a random effect PCM model.

- The mean of the latent trait distribution was set to 0 in the calibration sample, to reflect a perfect targeting between the sample and the PRO instrument.

- Variance of the latent trait distribution was set to 1 or 2 , to explore different cases of heterogeneity of the calibration population.

Trial samples varied between scenarios based on several parameters:

- The sample size within each treatment group, $N_{\text {trial}}$, varied between 50 and 500 (equal size between the two groups). Values were selected to reflect the range of sample sizes that can be encountered in clinical trials.

- The effect size of the treatment (standardized mean difference of patients' latent traits between treatment groups), $\gamma$, varied between 0 and 0.8 to explore various scenarios from no to large difference between treatment groups.

- The mean $\mu$ of the latent traits varied from 0 to 2.5 to explore cases were the trial sample and the PRO instrument showed perfect targeting to high mistargeting. Mistargeting may typically occur in practice when the trial population differs from the population of the validation study of the PRO instrument used for calibration (e.g., more or less severe sample with regards to the disease). Within treatment groups, the mean of latent traits was thus respectively $\mu_{0}$ for placebo and $\mu_{0}+\gamma$ for treatment group.

- Variance within each treatment group was set to 1. 
Each simulation scenario resulted in a set of PRO responses for a calibration sample and a trial sample and was replicated 500 times. Details of all simulation parameters with their possible values are described in Table 1. Data were simulated using the -simirt- module from STATA software. [26]

Table 1

Values of simulation parameters

\begin{tabular}{|c|c|c|}
\hline Characteristics & Parameter & Values \\
\hline \multirow{7}{*}{$\begin{array}{l}\text { PRO } \\
\text { instrument }\end{array}$} & Number of items $\mathrm{J}$ & $4,7,10$ \\
\hline & $\begin{array}{l}\text { Number of response categories } M \text { (response options } \\
\text { from } 0 \text { to } M-1 \text { ) }\end{array}$ & 3,5 \\
\hline & \multirow[t]{5}{*}{$\begin{array}{l}\text { Item locations }\left(\delta_{j}\right) \text { and category thresholds }\left(\delta_{j l}\right) \\
\text { distribution }\end{array}$} & $\begin{array}{l}\text { First archetype: } \\
\delta_{j}=-0.25+\frac{0.5}{J-1}(j-1), j=1 \ldots J \text { (Regularly spaced between } \\
-0.25+0.25)\end{array}$ \\
\hline & & $\begin{array}{l}\delta_{j l} \text { defined as the percentiles from a normal distribution with SD } \\
\text { of } 2.5\end{array}$ \\
\hline & & Second archetype: \\
\hline & & $\begin{array}{l}\delta_{j}=-1+\frac{2}{J-1}(j-1), j=1 \ldots J(\text { Regularly spaced between }-1 \\
+1)\end{array}$ \\
\hline & & $\begin{array}{l}\delta_{j l} \text { defined as the percentiles from a normal distribution with SD } \\
\text { of } 1.5\end{array}$ \\
\hline \multirow[t]{3}{*}{$\begin{array}{l}\text { Calibration } \\
\text { sample }\end{array}$} & Sample size $N_{\text {validation }}$ & $100,250,500$ \\
\hline & Variance & 1,2 \\
\hline & Mean of latent trait & 0 \\
\hline \multirow[t]{4}{*}{ Trial sample } & Sample size $N_{\text {trial }}$ & $50,100,200,500$ (per group) \\
\hline & $\begin{array}{l}\text { Effect size (Standardized mean difference between } \\
\text { groups) Y }\end{array}$ & $0,0.2,0.5$ \\
\hline & Mean of latent trait $\mu$ & $0,0.5,2$ \\
\hline & Variance within each group & 1 \\
\hline
\end{tabular}

Estimation

Simulated PRO data from each sample (calibration and trial) and within each scenario were analysed using a random effect PCM. A treatment group covariate (fixed effect) was also included in the model for the analysis of the trial samples (Equation 2). Treatment effect parameter ( $\mathrm{Y}$ ) and difficulties associated to category thresholds of each item $\left(\delta_{j l}\right)$ were estimated by maximizing the marginal likelihood $(\mathrm{MML})$. [27] In the trial samples, the estimators of each patient latent trait were also obtained using expected a posteriori Bayesian estimates. [18]

Calibration

The calibrated and non-calibrated approaches were used, for each scenario.

In the calibrated approach, item parameters were estimated based on the calibration sample. The obtained values for $\delta_{j l}$ were then assumed to be known without error and considered as fixed for the analysis of the trial sample.

In the non-calibrated approach, the calibration sample was not considered, and the estimation of item parameters was directly conducted on the trial sample.

Comparison of treatment groups

Two methods were used to compare treatment groups in the trial sample, for each simulated scenario, and for calibrated and non-calibrated approaches:

- Direct estimation of treatment group effect $\hat{\gamma}$, and testing of the nullity of the parameter using a Wald test.

$\wedge$

- Comparison of expected a posteriori Bayesian patient latent trait parameter $\theta_{i}$ between the treatment groups using a t-test. 
Criteria for comparison of approaches

The calibrated and non-calibrated approaches were compared along with the method used for comparing treatment groups based on the following criteria:

- Type-I error (a risk), which was obtained by computing the proportion of rejection of the null hypothesis among the 500 replications of each scenario with no simulated a priori difference between treatment groups $(Y=0)$.

- Power (1- $\beta$ ), which was obtained by computing the proportion of rejection of the null hypothesis among the 500 replications of each scenario with simulated a priori difference between treatment groups $(\gamma \neq 0)$.

- Position bias on the estimation of the treatment effect, which was obtained by computing the mean of the observed differences between $\hat{\gamma}$ and $\gamma$ based on the 500 replications of each scenario.

- Standard deviation of the estimate of treatment effect, which was obtained by computing the standard deviation of the obtained $\hat{\gamma}$ from the 500 replications of each scenario.

Analyses were performed using STATA software, version 14.

\section{Results}

Table 2 displays, for selected scenarios of interest, the results of the simulation study: type-I error, power, position bias and SD of the estimates for the difference between treatment groups. The scenarios were selected to focus on the parameters that showed an impact on any of these criteria, and to retain medium values for power, for a better interpretability of the results (to avoid a ceiling effect, i.e., a power of $100 \%$ ). The following 36 scenarios are presented: $\mathrm{J}$ of 4,7 or $10, \mathrm{M}$ of 3 or 5 , distribution of item parameters corresponding to the second archetype $(S D=1.5$, range $=2)$, $\mathrm{N}_{\text {calibration }}$ of 250 , variance of $1, \mathrm{~N}_{\text {trial }}$ of 200 or $500, \mu$ of $0,0.5$ or $2, y$ of 0.2 ( 0 for the calculation of type-l error). Comprehensive results for other scenarios can be found in supplementary materials (Additional file 1). Overall, the type-l error was well controlled and remained unchanged for all explored scenarios, i.e. calibration approaches and comparison of groups methods.

Impact of calibration

The simulations did not show any impact of the use of the calibration approach on the type-I error, power, position bias and SD of the estimates (Table 2). In particular, there was no impact even in the most disadvantageous cases for the calibration approach as compared to non-calibration (cases where the item parameters estimated from the calibration sample are expected to be less precise than the ones estimated from the trial): high mistargeting $\mu$, small $N_{\text {calibration }}$ and large $N_{\text {trial }}$, small variance of the calibration sample. There was thus no impact of the calibration sample parameters ( $N_{\text {calibration }}$ and variance of the sample) on any criteria. The absence of impact of the calibration approach is visible in the example scenario presented in Figure 2, as the power was similar for the calibrated and non-calibrated approaches (the curves overlap), for all levels of mistargeting.

Impact of comparison of treatment groups method

The simulations did not show any impact of the comparison of treatment groups method on the power and type-I error (Table 2). There was no position bias when estimating the treatment effect using a group covariate. However, a position bias was found when using patient latent trait estimates, the difference between groups being all the more underestimated as the number of items $\mathrm{J}$ was small and mistargeting was large. Of note, the SD of the estimates was higher when using a direct estimation of treatment group effect as compared to using a posteriori Bayesian patient latent trait estimates.

Impact of trial sample characteristics

As expected, an increase of the power with the sample size of the trial ( $N_{\text {trial }}$, see Table 2$)$ and the effect size $(\gamma$, data not shown) was observed. Results with a small mistargeting $(\mu=0.5)$ or optimal targeting $(\mu=0)$ resulted in comparable power. A large mistargeting of the sample $(\mu=2)$ resulted in a lower power (Figure 2 and Table 2).

Impact of PRO instrument characteristics

Increased number of items and response categories resulted in increased power (Figure 2 and Table 2). Also, the position bias observed using a posteriori Bayesian patient latent trait estimates was reduced when the number of items and response categories increased (Table 2). The number of item and response categories did not show any impact on the type-I error and the SD of the estimate of treatment effect (Table 2). The distribution of the item and response categories did not show any clear impact on any criteria.

Table 2. Type-l error, power, position bias and SD of the treatment effect estimates 
Legend: results are presented for selected scenarios, with $N_{\text {calibration }}=250$ distribution of the item parameters=second archetype with SD of 1.5 and range of 2 , and variance of the calibration sample $=1$. 


\begin{tabular}{|c|c|c|c|c|c|c|c|c|c|c|c|c|c|c|c|c|c|c|c|}
\hline \multirow[t]{5}{*}{$\mathrm{J}$} & \multirow[t]{5}{*}{$M$} & \multirow{5}{*}{$\mathrm{N}_{\text {trial }}$} & \multirow[t]{5}{*}{$\mu$} & \multirow{2}{*}{\multicolumn{4}{|c|}{$\begin{array}{l}Y=0 \\
\text { Type-l error }\end{array}$}} & \multicolumn{12}{|c|}{$Y=0.2$} \\
\hline & & & & & & & & \multicolumn{4}{|c|}{ Power } & \multicolumn{4}{|c|}{ Position bias } & \multicolumn{4}{|c|}{ SD of the estimates } \\
\hline & & & & \multicolumn{2}{|c|}{$\begin{array}{l}\text { Non } \\
\text { calibrated }\end{array}$} & \multicolumn{2}{|c|}{ Calibrated } & \multicolumn{2}{|c|}{$\begin{array}{l}\text { Non } \\
\text { calibrated }\end{array}$} & \multicolumn{2}{|c|}{ Calibrated } & \multicolumn{2}{|c|}{$\begin{array}{l}\text { Non } \\
\text { calibrated }\end{array}$} & \multicolumn{2}{|c|}{ Calibrated } & \multicolumn{2}{|c|}{$\begin{array}{l}\text { Non } \\
\text { calibrated }\end{array}$} & \multicolumn{2}{|c|}{ Calibrated } \\
\hline & & & & $\hat{\gamma}$ & $\begin{array}{l}\mathrm{t} \\
\text { test } \\
\text { on }\end{array}$ & $\hat{\gamma}$ & $\begin{array}{l}\mathrm{t} \\
\text { test } \\
\text { on }\end{array}$ & $\hat{\gamma}$ & $\begin{array}{l}\mathrm{t} \\
\text { test } \\
\text { on }\end{array}$ & $\hat{\gamma}$ & $\begin{array}{l}\mathrm{t} \\
\text { test } \\
\text { on }\end{array}$ & $\hat{\gamma}$ & $\begin{array}{l}\mathrm{t} \\
\text { test } \\
\text { on }\end{array}$ & $\hat{\gamma}$ & $\begin{array}{l}\mathrm{t} \\
\text { test } \\
\text { on }\end{array}$ & $\hat{\gamma}$ & $\begin{array}{l}\mathrm{t} \\
\text { test } \\
\text { on }\end{array}$ & $\hat{\gamma}$ & $\begin{array}{l}\mathrm{t} \\
\text { test } \\
\text { on }\end{array}$ \\
\hline & & & & & $\theta_{i}$ & & $\theta_{i}$ & & $\theta_{i}$ & & $\theta_{i}$ & & $\theta_{i}$ & & $\theta_{i}$ & & $\theta_{i}$ & & $\theta_{i}$ \\
\hline \multirow[t]{12}{*}{4} & \multirow[t]{6}{*}{3} & 200 & 0 & 5.6 & 5.6 & 5.6 & 5.6 & 32.8 & 32.8 & 32.8 & 32.6 & 0.01 & 0.08 & 0.01 & 0.08 & 0.13 & 0.08 & 0.13 & 0.08 \\
\hline & & & 0.5 & 4.4 & 4.4 & 4.6 & 4.4 & 32.8 & 32.8 & 33.2 & 33.0 & 0.00 & 0.08 & 0.00 & 0.08 & 0.13 & 0.08 & 0.13 & 0.08 \\
\hline & & & 2 & 5.8 & 5.6 & 5.6 & 5.4 & 29.6 & 29.6 & 29.8 & 29.6 & 0.00 & 0.10 & 0.01 & 0.10 & 0.15 & 0.07 & 0.15 & 0.07 \\
\hline & & 500 & 0 & 6.8 & 6.6 & 6.8 & 6.8 & 68.0 & 68.0 & 68.0 & 68.0 & 0.00 & 0.08 & 0.00 & 0.08 & 0.08 & 0.05 & 0.08 & 0.05 \\
\hline & & & 0.5 & 2.6 & 2.6 & 2.6 & 2.6 & 68.4 & 68.4 & 68.6 & 68.4 & 0.00 & 0.08 & 0.00 & 0.08 & 0.08 & 0.05 & 0.08 & 0.05 \\
\hline & & & 2 & 4.6 & 4.6 & 4.6 & 4.6 & 58.6 & 58.6 & 58.4 & 58.4 & 0.00 & 0.11 & 0.00 & 0.10 & 0.09 & 0.04 & 0.09 & 0.05 \\
\hline & 5 & 200 & 0 & 4.6 & 4.6 & 4.8 & 4.6 & 41.2 & 41.2 & 41.2 & 40.6 & 0.00 & 0.05 & 0.00 & 0.05 & 0.12 & 0.09 & 0.12 & 0.09 \\
\hline & & & 0.5 & 4.6 & 4.6 & 4.6 & 4.4 & 40.8 & 40.8 & 40.6 & 40.4 & 0.00 & 0.05 & 0.00 & 0.05 & 0.12 & 0.09 & 0.12 & 0.09 \\
\hline & & & 2 & 5.4 & 5.4 & 5.6 & 5.4 & 32.2 & 32.4 & 32.6 & 32.4 & 0.00 & 0.08 & 0.00 & 0.07 & 0.13 & 0.08 & 0.13 & 0.08 \\
\hline & & 500 & 0 & 5.2 & 5.2 & 5.2 & 5.2 & 76.6 & 76.6 & 76.8 & 76.6 & 0.00 & 0.05 & 0.00 & 0.05 & 0.07 & 0.06 & 0.07 & 0.06 \\
\hline & & & 0.5 & 4.6 & 4.6 & 4.6 & 4.6 & 75.6 & 75.6 & 75.8 & 75.8 & 0.00 & 0.05 & 0.00 & 0.05 & 0.08 & 0.06 & 0.08 & 0.06 \\
\hline & & & 2 & 4.2 & 4.2 & 4.2 & 4.2 & 69.8 & 69.8 & 69.4 & 69.4 & 0.00 & 0.08 & 0.00 & 0.07 & 0.08 & 0.05 & 0.08 & 0.05 \\
\hline 7 & 3 & 200 & 0 & 7.6 & 7.2 & 7.4 & 7.2 & 40.8 & 40.8 & 40.8 & 40.8 & 0.00 & 0.05 & 0.00 & 0.05 & 0.12 & 0.09 & 0.12 & 0.09 \\
\hline & & & 0.5 & 5.4 & 5.2 & 5.6 & 5.2 & 40.0 & 40.0 & 40.2 & 40.0 & 0.00 & 0.06 & 0.00 & 0.06 & 0.12 & 0.08 & 0.12 & 0.08 \\
\hline & & & 2 & 5.2 & 5.2 & 5.2 & 5.2 & 36.6 & 36.6 & 36.6 & 36.4 & 0.01 & 0.08 & 0.01 & 0.08 & 0.13 & 0.08 & 0.13 & 0.08 \\
\hline & & 500 & 0 & 5.2 & 5.2 & 5.2 & 5.2 & 76.8 & 76.6 & 77.0 & 76.8 & 0.00 & 0.05 & 0.00 & 0.05 & 0.07 & 0.05 & 0.07 & 0.05 \\
\hline & & & 0.5 & 5.2 & 5.2 & 5.2 & 5.2 & 75.0 & 75.0 & 75.0 & 75.0 & 0.00 & 0.05 & 0.00 & 0.05 & 0.08 & 0.06 & 0.08 & 0.06 \\
\hline & & & 2 & 3.6 & 3.6 & 3.6 & 3.6 & 67.8 & 67.8 & 67.8 & 67.8 & 0.00 & 0.08 & 0.00 & 0.08 & 0.08 & 0.05 & 0.09 & 0.05 \\
\hline & 5 & 200 & 0 & 5.2 & 5.2 & 5.2 & 5.2 & 42.6 & 42.6 & 42.8 & 42.6 & 0.00 & 0.03 & 0.00 & 0.03 & 0.11 & 0.09 & 0.11 & 0.09 \\
\hline & & & 0.5 & 5.2 & 5.0 & 5.2 & 5.0 & 46.0 & 45.6 & 46.0 & 46.0 & 0.00 & 0.03 & 0.00 & 0.03 & 0.11 & 0.10 & 0.11 & 0.10 \\
\hline & & & 2 & 6.4 & 6.4 & 6.4 & 6.4 & 41.2 & 40.6 & 41.2 & 40.6 & 0.00 & 0.05 & 0.01 & 0.05 & 0.12 & 0.09 & 0.12 & 0.09 \\
\hline & & 500 & 0 & 6.8 & 6.8 & 6.8 & 6.8 & 80.8 & 80.8 & 80.8 & 80.6 & 0.00 & 0.03 & 0.00 & 0.03 & 0.07 & 0.06 & 0.07 & 0.06 \\
\hline & & & 0.5 & 3.2 & 3.0 & 3.2 & 3.2 & 80.8 & 80.8 & 80.6 & 80.6 & 0.00 & 0.03 & 0.00 & 0.03 & 0.07 & 0.06 & 0.07 & 0.06 \\
\hline & & & 2 & 5.2 & 5.2 & 5.4 & 5.2 & 73.6 & 73.6 & 73.4 & 73.2 & 0.01 & 0.06 & 0.01 & 0.06 & 0.07 & 0.05 & 0.08 & 0.06 \\
\hline 10 & 3 & 200 & 0 & 4.2 & 4.2 & 4.2 & 4.2 & 42.6 & 42.4 & 42.6 & 42.2 & 0.00 & 0.04 & 0.00 & 0.04 & 0.11 & 0.09 & 0.12 & 0.09 \\
\hline & & & 0.5 & 5.2 & 5.2 & 5.2 & 5.2 & 42.0 & 41.6 & 42.2 & 41.6 & 0.00 & 0.04 & 0.00 & 0.04 & 0.11 & 0.09 & 0.11 & 0.09 \\
\hline & & & 2 & 4.0 & 3.6 & 4.0 & 3.6 & 39.2 & 39.0 & 39.2 & 39.0 & 0.00 & 0.07 & 0.00 & 0.07 & 0.13 & 0.08 & 0.13 & 0.09 \\
\hline & & 500 & 0 & 4.8 & 4.4 & 4.6 & 4.4 & 79.4 & 79.2 & 79.4 & 79.2 & 0.00 & 0.04 & 0.00 & 0.04 & 0.07 & 0.06 & 0.07 & 0.06 \\
\hline & & & 0.5 & 6.2 & 6.2 & 6.2 & 6.2 & 78.8 & 78.8 & 78.8 & 78.8 & 0.00 & 0.04 & 0.00 & 0.04 & 0.07 & 0.06 & 0.07 & 0.06 \\
\hline & & & 2 & 8.2 & 8.2 & 8.2 & 8.2 & 76.0 & 75.8 & 75.8 & 75.6 & 0.00 & 0.07 & 0.00 & 0.06 & 0.08 & 0.05 & 0.08 & 0.05 \\
\hline & 5 & 200 & 0 & 5.0 & 5.0 & 5.2 & 5.0 & 46.0 & 45.8 & 45.4 & 45.4 & 0.00 & 0.02 & 0.00 & 0.02 & 0.10 & 0.09 & 0.10 & 0.09 \\
\hline & & & 0.5 & 5.6 & 5.6 & 6.0 & 5.6 & 47.2 & 46.8 & 47.2 & 46.4 & 0.00 & 0.02 & 0.00 & 0.02 & 0.11 & 0.09 & 0.11 & 0.10 \\
\hline & & & 2 & 4.4 & 4.2 & 4.4 & 4.0 & 42.0 & 41.6 & 42.0 & 41.6 & 0.00 & 0.04 & 0.00 & 0.04 & 0.11 & 0.09 & 0.11 & 0.09 \\
\hline & & 500 & 0 & 5.6 & 5.4 & 5.6 & 5.6 & 84.4 & 84.2 & 84.4 & 84.2 & 0.00 & 0.02 & 0.00 & 0.02 & 0.07 & 0.06 & 0.07 & 0.06 \\
\hline & & & 0.5 & 6.2 & 6.2 & 6.2 & 6.2 & 83.8 & 83.8 & 84.2 & 83.8 & 0.00 & 0.02 & 0.00 & 0.02 & 0.07 & 0.06 & 0.07 & 0.06 \\
\hline
\end{tabular}




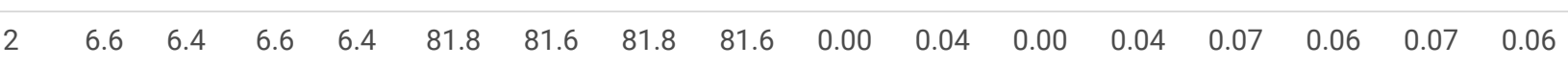

\section{Discussion}

This simulation study explored the impact of calibration of polytomous PRO instruments on the comparison of treatment groups in a clinical trial. This impact was evaluated within the RMT framework, considering different methods for comparison of treatment groups, and various settings (characteristics of the PRO instrument, calibration and trial samples). The lack of impact of calibration observed in the study showed that the benefit in terms of interpretability, brought by the traceability property warranted by calibration, is not obtained at the expense of the ability to show a true difference between treatment groups or in terms of proper control of the type-l error. Given its important added value, calibration should thus always be performed when a PRO instrument is used as an endpoint in a clinical trial, in the RMT framework.

The simulations consistently showed that the type-l error, power of test for the comparison of the two groups, bias, and dispersion of the estimated difference between treatment groups were similar for calibrated and non-calibrated approaches. Calibration did not have any impact even in the most favorable cases for the use of non-calibrated estimates, i.e., when the calibration sample size was small with low variance and when the trial sample size was large with high mistargeting. The present results also confirmed previous simulation studies. Blanchin et al. explored the impact of misspecification of dichotomous item parameters at the design stage, while attempting to estimate the power of a clinical trial. [20] They showed that such misspecification had no impact on power, which indirectly support calibration: errors in the item parameters used for calibration would not likely impact power. [20] Findings were also consistent with simulation studies from Sébille et al. and Hamel et al., which included comparison of cases where the dichotomous item parameters were considered as known (i.e. use of calibration) or unknown and estimated from the trial data (noncalibration). [19, 28]

Regardless of the calibration situation, mistargeting of the PRO instrument to the clinical trial sample impacted the ability to detect a treatment effect in the clinical trial. Indeed, a large mistargeting of the sample resulted in lower power and higher dispersion of the estimates of the treatment effect. This is consistent with the findings from a previous simulation study, where mistargeting between the PRO instrument (with dichotomous items) and the sample was associated to lower power. [29] This confirms that PRO instruments should be properly targeted to the level of severity of the patient population included in the trial, to be able to effectively detect treatment effect. This is especially true when the mistargeting results in floor or ceiling effect (i.e., when no items are included to capture low or high level of the measured concept), as it was the case in this work for the scenarios with large mistargeting. A small mistargeting did not seem to impact the results, but this should be interpreted cautiously, as it may be affected by the exact distribution of the items and patients over the continuum: lower levels of mistargeting might still show an impact when item distribution is very uneven or associated to less homogenous or non-normally distributed patient samples.

Additionally, and as already flagged by multiple studies, higher number of items and response categories resulted in higher power. [19, 28] This impact on power can be compared to the one, well known, of the number of patients included in the trial. Considering the case of a trial with 200 patients with an effect size of 0.2 , our simulations showed that shifting from 4 items with 3 response categories to 10 items with 5 response categories represented an increase of power from $30-45 \%$. Considering the same example case and the simulation results, this is approximately similar to the impact on power that would be observed from adding 100 patients to the trial. This confirms the importance of using PRO instruments that include enough items in small sample studies. This aspect should be carefully considered when shorter instruments are recommended, typically to "minimize patients' response burden". [30] Also, interestingly, the decrease of power due to a high mistargeting was lower when the PRO instruments included a large number of items and response categories (note that, as noted above, this finding may be somewhat dependent on the specific distribution of items and patients used in our simulations).

This study came with several limitations and further necessary developments can be underlined. First, the calibration process only investigated the case where patients only differed based on their level of latent trait between the calibration and the trial sample. But in real-life studies, patients can differ on other characteristics, such as their demographics, etc. In some cases, these characteristics impact the way patients respond to the items, despite having the same level of latent trait: item parameter values may differ depending on these characteristics, which is known as differential item functioning (DIF). [31] If patients from the calibration and the trial sample differ based on a characteristic that creates DIF (e.g., they have different disease subtypes, or different countries imply cultural differences despite having the same language, etc.), the item parameter values used from the calibration sample will not be fully adequate for the clinical trial. A solution may be to obtain different sets of item parameters values from different calibration samples, to be used alternatively to calibrate the measure depending on the population of the trial. For example, different sets of calibration are proposed to calculate PROMIS scores. [32] But in many cases when conducting a clinical trial, there is no available calibration set perfectly suited to the population of interest. In this situation, it is possible that calibration with wrongly specified parameters would hinder the ability of the trial to accurately assess an effect of the treatment. A previous simulation study showed that DIF, if ignored in the analysis, could result in biased estimates of the difference between groups. [33,34] The impact of calibration in the presence of DIF should thus be further explored. Another limitation stands in the approaches for comparison of treatment groups that were explored in this study. Our simulations only considered results from a random effect PCM. Considering statistical methods that compare individual PRO measures in a different estimation context would be informative. Typically, investigating the implication of using statistical methods that compare PRO estimates from a fixed effect PCM with pairwise conditional maximum likelihood (as performed in RUMM, one of the currently most commonly used software for RMT analysis [35]) would allow

Page $9 / 14$ 
gaining a better understanding on the various options for the analysis of PRO measures resulting from a RMT paradigm in a clinical trial, and the relative impact of calibration in these various cases. Also, since the distributions used within this simulation study were normal and with the patient data showing an optimal fit to the model, the performance of a random effect PCM might have been overestimated as compared to analysis on real observed data. Overall, further studies using non-normal simulated PRO data, or with a non-optimal fit to the Rasch model would also be of interest. Finally, we deliberately restricted the scope of these analyses to the RMT framework, because of its key property of specific objectivity, which is an essential property for calibration and from a metrological perspective in general. Future research could explore whether our conclusions are confirmed in the context of calibration in an IRT paradigm.

This work showed that calibration was always an appropriate option when analysing PRO endpoints from a clinical trial. For calibration to be possible, the PRO instrument must have previously undergone RMT analysis, with a set of item parameters available in the literature (set of values to be re-used in different trials). Some instruments developed in the RMT paradigm provide the possibility to calibrate the estimates in other studies, such as the BREAST-Q [14] and other instruments from the Q-portfolio, the R-ODS [15], the StomaQoL [36] or the 88-item Multiple Sclerosis Spasticity Scale (MSSS-88) [37]. Similarly, the PROMIS or the European Organization for Research and Treatment of Cancer Quality of Life Questionnaire (EORTC QLQ-C30) computerized adaptative testing (CAT) also use calibration, but in an IRT paradigm. [16, 38] However, it does not seem to be systematically the case. $[39,40]$ Based on the findings of our simulations, we would recommend that calibration is consistently considered by developers of new PRO instruments using the RMT framework, and by clinical trial statisticians who are analysing data from these instruments. Using a formal RMT analysis, the treatment groups can be compared by including a covariate in a random effect Rasch model. Patient latent traits can also be estimated, based on a random or fixed effect Rasch model, before comparing the groups (e.g., using a t-test). Another, simpler option to obtain calibrated measures of patient latent traits is to use conversion tables that allows transforming raw scores to approximated measurements from the Rasch model. Shortcomings of this approach include that patient measurements cannot be assessed in the presence of missing item. While these different methods allowing for calibration seem to perform differently (e.g., performing a t-test on patient estimated latent traits from a random effect Rasch model showed to be biased, as observed in the current study [19]), there is no definitive consensus on the method to be preferred. The methods used will also have to be carefully considered to appropriately take benefit of the metrological advantages of the PRO measures underpinned by the Rasch model (i.e., possibility of having interval-level scales and measurement uncertainty at the individual level).

\section{Conclusions}

The RMT framework allows for proper calibration of PRO instruments in clinical trials. In such context, our simulation study showed that calibration of the PRO instruments should be consistently performed since it guarantees interpretability of the results, through traceability, while still showing similar ability of the trial to demonstrate treatment effect. For calibration to be possible, proper sets of item parameters values or conversion tables obtained from calibration samples should be provided for the PRO instruments developed with RMT.

\section{Abbreviations}

CAT, Computerized adaptative testing; CTT, Classical test theory; DIF, Differential item functioning; EORTC QLQ-C30, European Organization for Research and Treatment of Cancer Quality of Life Questionnaire; IRT, Item response theory; MML, Marginal maximum likelihood; MSSS-88, 88-item Multiple Sclerosis Spasticity Scale; PCM, Partial Credit Model; PRO, Patient-reported outcome; PROMIS, PRO Measurement Information System; RMT, Rasch measurement theory; R-ODS, Rasch-built overall disability scale; SAT, Scholastic aptitude test; SD, Standard deviation.

\section{Declarations}

\section{Ethics approval and consent to participate}

Not applicable

\section{Consent for publication}

Not applicable

\section{Availability of data and materials}

The datasets generated and analysed during the current study are available in the OSF repository, https://osf.io/prbaj/? view_only=9c7493c7ba6548bf9819e4d730077b3e. The Stata ${ }^{\odot}$ programs used to analyse the datasets are available from the corresponding author.

\section{Competing interests}

The authors declare that they have no competing interest.

\section{Funding}

The work was supported by the French "Association nationale de la recherche et de la technologie" (ANRT) funding under CIFRE PhD program to AL. 


\section{Authors' contributions}

$\mathrm{AL}, \mathrm{AR}$, VS and JBH conceived the study, interpreted the results, and drafted the manuscript. AL performed the simulation study. All authors read and approved the final manuscript.

\section{Acknowledgements}

The authors want to thank Stefan Cano for his insight on previous versions of this manuscript.

\section{References}

1. Health, U.D.o. and H. Services, Guidance for industry-Patient-reported outcome measures: Use in medical product development to support labeling claims. https://www.fda.gov/media/77832/download, 2009.

2. Mercieca-Bebber, R., et al., The importance of patient-reported outcomes in clinical trials and strategies for future optimization. Patient related outcome measures, 2018. 9: p. 353.

3. Maul, A., et al., The quality of measurement results in terms of the structural features of the measurement process. 2018. 116: p. 611-620.

4. Fisher Jr, W.P.J.M., Invariance and traceability for measures of human, social, and natural capital: Theory and application. 2009. 42(9): p. 12781287.

5. Cano, S., et al., Towards consensus measurement standards for patient-centered outcomes. Measurement, 2019. 141: p. 62-69.

6. De Bièvre, P.J.A. and Q. Assurance, The 2012 International Vocabulary of Metrology: “VIM”. 2012. 17(2): p. $231-232$.

7. Phillips, S.D., et al., A careful consideration of the calibration concept. Journal of research of the National Institute of Standards and Technology, 2001. 106(2): p. 371.

8. An, X. and Y.-F. Yung, Item response theory: What it is and how you can use the IRT procedure to apply it. SAS Institute Inc. SAS364-2014, 2014. 10(4).

9. Nunnally Jr, J.C., Introduction to psychological measurement. 1970.

10. Wright, B.D., J.M.J.A.o.p.m. Linacre, and rehabilitation, Observations are always ordinal; measurements, however, must be interval. 1989. 70(12): p. 857-860.

11. Rasch, G.J.C.D.I.f.E.R., Probabilistic models for some intelligence and achievement tests. 1960. 56.

12. Andrich, D., Rasch models for measurement. Vol. 68. 1988: Sage.

13. Hobart, J. and S. Cano, Improving the evaluation of therapeutic interventions in multiple sclerosis: the role of new psychometric methods. 2009: Prepress Projects.

14. Pusic, A.L., et al., Development of a new patient-reported outcome measure for breast surgery: the BREAST-Q. Plastic and reconstructive surgery, 2009. 124(2): p. 345-353.

15. van Nes, S.I., et al., Rasch-built Overall Disability Scale (R-ODS) for immune-mediated peripheral neuropathies. Neurology, 2011. 76(4): p. 337345.

16. DeWalt, D.A., et al., Evaluation of item candidates: the PROMIS qualitative item review. Medical care, 2007. 45(5 Suppl 1): p. S12.

17. Christensen, K.B., et al., Latent regression in loglinear Rasch models. Communications in Statistics-Theory and Methods, 2004. 33(6): p. 12951313.

18. Hoijtink, H. and A. Boomsma, On person parameter estimation in the dichotomous Rasch model, in Rasch Models. 1995, Springer. p. 53-68.

19. Hamel, J.-F., et al., Biases and power for groups comparison on subjective health measurements. 2012. 7(10): p. e44695.

20. Blanchin, M., et al., Power and sample size determination for the group comparison of patient-reported outcomes using the Rasch model: impact of a misspecification of the parameters. BMC medical research methodology, 2015. 15(1): p. 21.

21. Rasch, G., Studies in mathematical psychology: I. Probabilistic models for some intelligence and attainment tests. 1960.

22. Andrich, D.J.P., A rating formulation for ordered response categories. 1978. 43(4): p. 561-573.

23. McGuigan, C. and M. Hutchinson, Confirming the validity and responsiveness of the Multiple Sclerosis Walking Scale-12 (MSWS-12). Neurology, 2004. 62(11): p. 2103-2105.

24. Lord, F.M., Applications of item response theory to practical testing problems. 2012: Routledge.

25. Anthoine, E., et al., Sample size used to validate a scale: a review of publications on newly-developed patient reported outcomes measures. Health and quality of life outcomes, 2014. 12(1): p. 1-10.

26. Hardouin, J.-B., SIMIRT: Stata module to process data generated by IRT models. 2013.

27. Molenaar, I.W., Estimation of item parameters, in Rasch models. 1995, Springer. p. 39-51.

28. Sébille, V., et al., Methodological issues regarding power of classical test theory (CTT) and item response theory (IRT)-based approaches for the comparison of patient-reported outcomes in two groups of patients-a simulation study. BMC medical research methodology, 2010. 10(1): $\mathrm{p}$. 1- 
10.

29. Blanchin, M., et al., Power and sample size determination for the group comparison of patient-reported outcomes with rasch family models. PLoS One, 2013. 8(2): p. e57279.

30. Food, U. and D. Administration, Value and use of Patient-Reported Outcomes (PROs) in assessing effects of medical devices. 2018.

31. Thissen, D., L. Steinberg, and H. Wainer, Detection of differential item functioning using the parameters of item response models. 1993.

32. Reeve, B.B., et al., Psychometric evaluation and calibration of health-related quality of life item banks: plans for the Patient-Reported Outcomes Measurement Information System (PROMIS). Medical care, 2007: p. S22-S31.

33. Rouquette, A., J.-B. Hardouin, and J. Coste, Differential Item Functioning (DIF) and subsequent bias in group comparisons using a composite measurement scale: A simulation study. J Appl Meas, 2016. 17: p. 312-334.

34. Rouquette, A., et al., Differential Item Functioning (DIF) in composite health measurement scale: Recommendations for characterizing DIF with meaningful consequences within the Rasch model framework. PloS one, 2019. 14(4): p. e0215073.

35. Andrich, D., B. Sheridan, and G. Luo, RUMM2030: Rasch unidimensional models for measurement. Perth, Western Australia: RUMM Laboratory, 2009.

36. Prieto, L., H. Thorsen, and K. Juul, Development and validation of a quality of life questionnaire for patients with colostomy or ileostomy. Health and Quality of Life Outcomes, 2005. 3(1): p. 1-10.

37. Hobart, J., et al., Getting the measure of spasticity in multiple sclerosis: the Multiple Sclerosis Spasticity Scale (MSSS-88). Brain, 2006. 129(1): p. 224-234.

38. Petersen, M.A., et al., Development of computerised adaptive testing (CAT) for the EORTC QLQ-C30 dimensions-general approach and initial results for physical functioning. European Journal of Cancer, 2010. 46(8): p. 1352-1358.

39. Belvedere, S.L. and N.A. de Morton, Application of Rasch analysis in health care is increasing and is applied for variable reasons in mobility instruments. Journal of clinical epidemiology, 2010. 63(12): p. 1287-1297.

40. de Bock, E., P. Williams, and B. Tugaut, Four decades of Rasch analysis on patient-reported outcomes instruments validation: A systematic literature review. Value in Health, 2016. 19(7): p. A362.

\section{Figures}


first archetype $(\mathrm{SD}=2.5$, range=0.5)
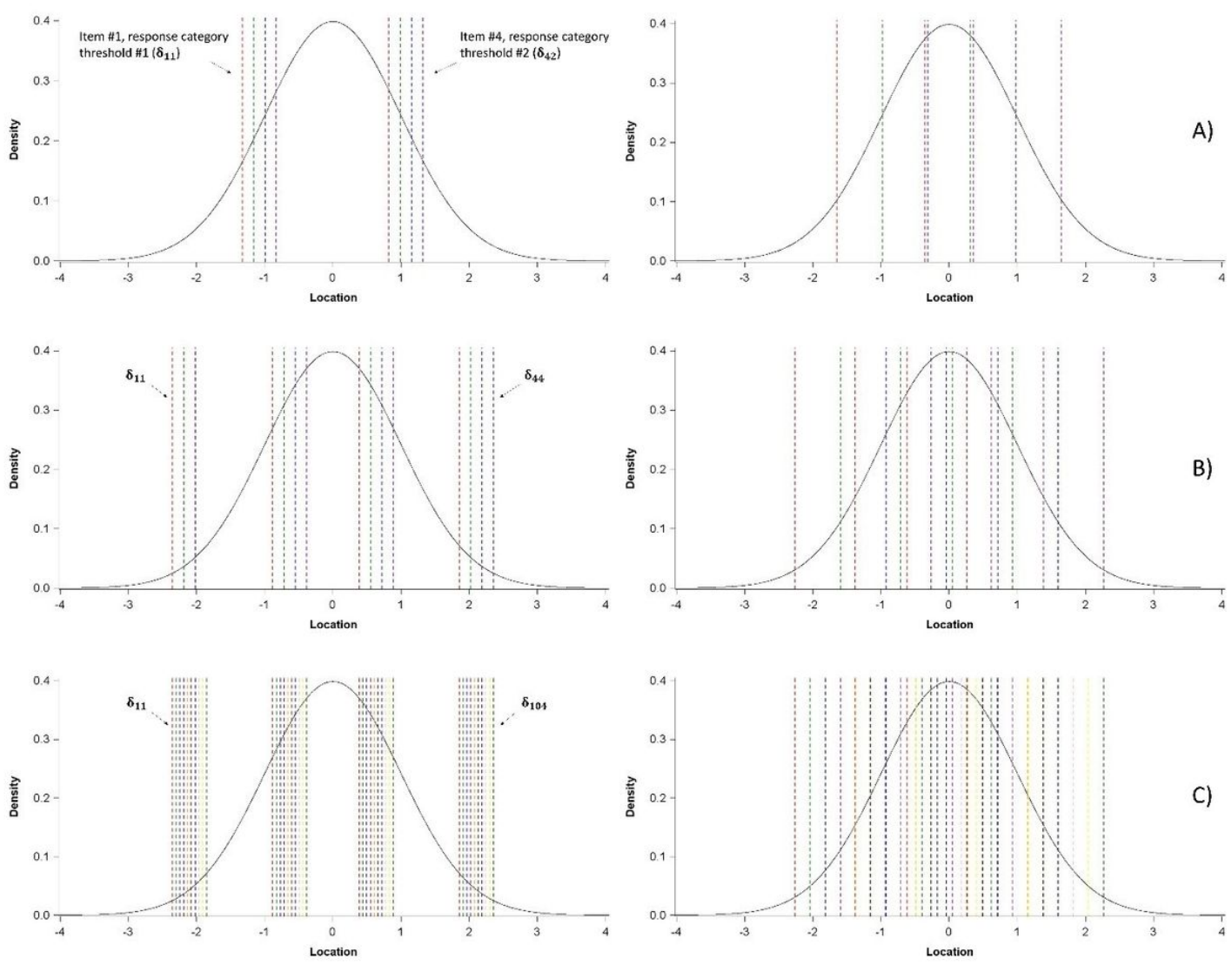

Figure 1. Illustration of the archetypes of items distribution, for different scenarios

Legend: Vertical dashed lines represent the item response category thresholds ( $\delta_{j l}$, with each color corresponding to a different item) in different scenarios, and the probability density function curve represents the distribution of the latent trait in the calibration sample (case with a variance $=1$ ) The left part of the figure includes cases where the item locations $\delta_{j}$ have a low dispersion (range $=0.5$ ) and the $\delta_{i l}$ have a high dispersion

$\left(\mathrm{SD}=2.5\right.$ ). The right part of the figure includes cases where the item locations $\delta_{j}$ have a high dispersion (range $=2$ ) and the $\delta_{j l}$ have a low
dispersion ( $(\mathrm{SD}=1.5$ ). Each line corresponds to different scenarios regarding the number of item and modalities: $\mathrm{A}) \mathrm{J}=4$ items, M=3 modalities. B) dispersion $(S D=1.5)$. Each line corresponds to different scen
$\mathrm{J}=4$ items, $M=5$ modalities. C) $\mathrm{J}=10$ items, $\mathrm{M}=5$ modalities.

\section{Figure 1}

"See image above for figure legend" 


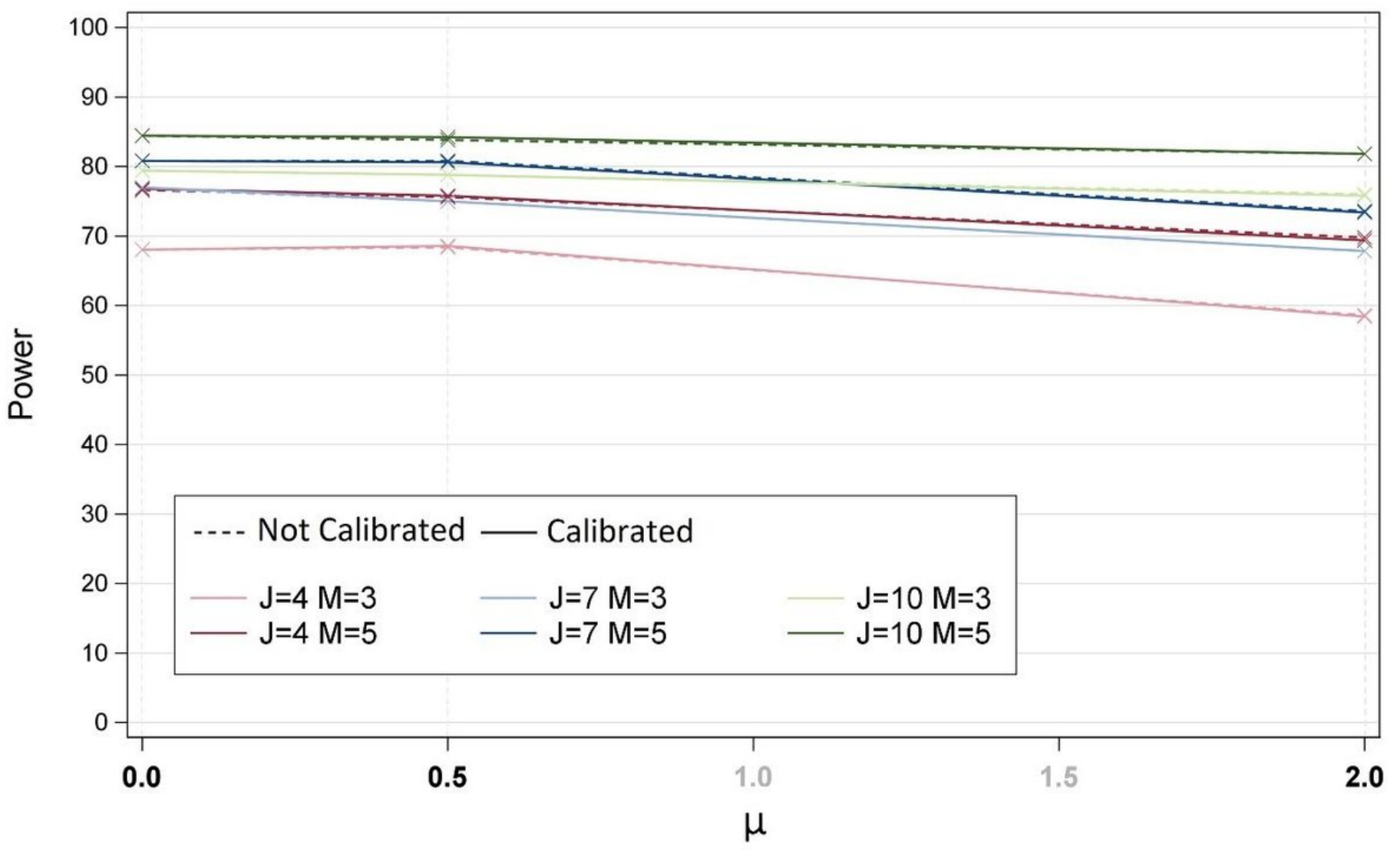

Figure 2. Power using calibrated and non-calibrated approaches, depending on mistargeting of the trial sample $\mu$

Legend: power is presented for instruments with varying number of items $\mathrm{J}$ and modalities $\mathrm{M}$. Presented results are for comparison of treatment groups based on $\hat{\gamma}$, for scenarios with the distribution of the item parameters=second archetype with SD of 1.5 and range of $2, \nu=0.2, N_{\text {trial }}=500$, $N_{\text {calibration }}=250$, variance of the calibration sample $=1$.

\section{Figure 2}

"See image above for figure legend"

\section{Supplementary Files}

This is a list of supplementary files associated with this preprint. Click to download.

- Additionalfile1.docx 